\title{
Prognostic relevance of cortical superficial siderosis in cerebral amyloid angiopathy
}

Frank Arne Wollenweber, MD, * Christian Opherk, MD,* Marialuisa Zedde, MD, Cihan Catak, MD, Rainer Malik, PhD, Marco Duering, MD, Marek Janusz Konieczny, MSc, Rosario Pascarella, MD, Raquel Samões, MD, Manuel Correia, PhD, Joan Martí-Fàbregas, MD, Jennifer Linn, MD, † and Martin Dichgans, MD†

Neurology ${ }^{\circledR}$ 2019;92:e792-e801. doi:10.1212/WNL.0000000000006956

\section{Abstract}

\section{Objective}

To investigate the prognostic relevance of cortical superficial siderosis (cSS) in patients with cerebral amyloid angiopathy (CAA).

\section{Methods}

A total of 302 patients fulfilling clinical and imaging criteria for probable or possible CAA were enrolled into a prospective, multicenter cohort study and followed for 12 months. cSS was assessed on $\mathrm{T}_{2}^{*} /$ susceptibility-weighted imaging MRI. The predefined primary composite endpoint was incident stroke or death in patients with cSS compared to those without. Secondary analyses included cerebrovascular events and functional outcome measured by the modified Rankin Scale (mRS). Multiple regression analysis was performed to adjust for possible confounders.

\section{Results}

cSS prevalence was $40 \%$. The primary endpoint occurred more frequently in patients with cSS $(22 \%, 27 / 121)$ compared to those without $(8 \%, 15 / 181, p=0.001)$. Rates of CAA-related incident intracranial hemorrhage were $17 \%$ (cSS) and $4 \%$ (no cSS, $p=0.0003$ ). The proportion of patients being functionally independent (mRS 0-2) 12 months from baseline were 59\% (cSS) and $82 \%$ (no cSS, $p=0.00002$ ). Presence of cSS was associated with the primary endpoint (adjusted odds ratio [OR] 1.2, 95\% confidence interval [CI] 1.1-1.3, $p=0.0005$ ), incident intracranial hemorrhage (adjusted OR 1.2, 95\% CI 1.1-1.3, $p=0.0003$ ), and less favorable outcome as assessed by the mRS (common OR 1.9, 95\% CI 1.2-3.1, $p=0.009$ ). Similar results were obtained in analyses restricted to patients with probable CAA and to patients with disseminated cSS (all $p<0.005$ ).

\section{Conclusions}

Patients with cSS and suspected CAA are at high risk for CAA-related incident intracranial hemorrhage and poor functional outcome. Both the presence and extent of cSS have prognostic relevance and may influence clinical decision-making.
Correspondence

Dr. Wollenweber

frank.wollenweber@

med.uni-munchen.de

\section{RELATED ARTICLE}

Editorial

Implications of cortical superficial siderosis in CAA: Superficial relationships

Page 360

*These authors contributed equally to this work as first authors.

tThese authors contributed equally to this work as last authors.

From the Institute for Stroke and Dementia Research (F.A.W., C.O., C.C., R.M., M. Duering, M.J.K., M. Dichgans), University Hospital, LMU Munich; Klinik für Neurologie (C.O.), SLK-Kliniken Heilbronn GmbH, Heilbronn, Germany; Stroke Unit-Neurology Unit and Neuroradiology Unit (M.Z., R.P.), Arcispedale Santa Maria Nuova, Azienda Unità Sanitaria Locale-IRCCS Reggio Emilia, Italy; Serviço de Neurologia (R.S., M.C.), Centro Hospitalar do Porto, Hospital de Santo António, Portugal; Hospital de la Santa Creu i Sant Pau (J.M.-F.), Biomedical Research Institute Sant Pau, IIB Sant Pau, Spain; Institut und Poliklinik für Neuroradiologie (J.L.), Universitätsklinikum Carl Gustav Carus, Dresden; Department of Neuroradiology (J.L.), Klinikum der Universität München, Ludwig-Maximilians-Universität LMU, Munich; Munich Cluster for Systems Neurology (SyNergy) (M. Dichgans); and German Center for Neurodegenerative Diseases (DZNE, Munich) (M. Dichgans), Germany.

Go to Neurology.org/N for full disclosures. Funding information and disclosures deemed relevant by the authors, if any, are provided at the end of the article. 


\section{Glossary}

CAA = cerebral amyloid angiopathy; $\mathbf{C I}=$ confidence interval; $\mathbf{C M B}=$ cerebral microbleeds; $\mathrm{cSS}$ = cortical superficial siderosis; ICH = intracerebral hemorrhage; $\mathbf{M M S E}=$ Mini-Mental State Examination; $\mathbf{m R S}=$ modified Rankin Scale; OR = odds ratio; PVS = perivascular spaces; $\mathbf{Q C}=$ quality control; SAH = subarachnoid hemorrhage; SVD = small vessel disease; SWI = susceptibility-weighted imaging; $\mathbf{W M H}=$ white matter hyperintensities.

Cerebral amyloid angiopathy (CAA), caused by deposition of $\beta$-amyloid in the walls of leptomeningeal arteries, ${ }^{1}$ is remarkably common in the elderly. ${ }^{2}$ Its major clinical consequences include intracerebral hemorrhages (ICH), typically in lobar location, ${ }^{3}$ and cognitive impairment. ${ }^{4}$ CAA is the underlying etiology in up to $74 \%$ of nontraumatic lobar $\mathrm{ICH}^{2}$ with mortality rates ranging between $10 \%$ and $30 \%{ }^{5,6}$ Moreover, CAA-related ICH has a high recurrence risk $(\sim 10 \%$ per year $)$ even in the absence of oral anticoagulation. ${ }^{7}$ Meanwhile, the number of elderly patients with CAA with concomitant atrial fibrillation is growing, ${ }^{8}$ thus posing a challenge to balancing the benefits and risks of antithrombotic treatment.

Recent single-center studies have suggested a prognostic relevance of cortical superficial siderosis (cSS) in predicting ICH risk in patients with probable or possible CAA. ${ }^{9-11}$ However, data from prospective, multicenter studies are missing, as are data on functional outcome and on incident events other than $\mathrm{ICH}$.

In this prospective, registered, multicenter cohort study, we set out to determine the prognostic value of cSS in patients with probable or possible CAA. Specifically, we aimed to compare the rate of incident stroke or death of any cause in patients with cSS compared to those without cSS. ${ }^{1}$ Secondary endpoints included the rate of incident intracranial hemorrhage (including both ICH and sulcal subarachnoid hemorrhage $[\mathrm{SAH}]$ ) and the proportion of patients being functionally independent 12 months from baseline.

\section{Methods}

\section{Study setting}

All data are derived from the Superficial Siderosis in Patients with Suspected Cerebral Amyloid Angiopathy Study (SuSPect-CAA), a prospective, hospital-based, multicenter, cohort study conducted at 4 university hospitals in Europe (Munich, Germany [coordinating center]; Reggio Emilia, Italy; Barcelona, Spain; and Porto, Portugal).

\section{Participants}

A total of 302 patients were enrolled into the study between May 2013 and March 2016. Patients meeting a diagnosis of probable or possible CAA based on the modified Boston criteria $^{12}$ were included with the following additional modification: we did not exclude patients with a maximum of 2 deep cerebral microbleeds (CMB) on baseline MRI provided they had at least twice the number of lobar CMB or a history of lobar ICH, or cSS as assessed by a central MRI read in consensus by 2 expert raters (F.A.W. and J.L.). This additional modification was made to avoid a potential selection bias towards CAA patients without hypertension. Major exclusion criteria were a history of $\mathrm{ICH}$ located in the deep gray matter (basal ganglia or thalami) or brainstem, history of head trauma resulting in loss of consciousness or radiologically visible brain injury in the last 5 years prior to inclusion, history of cerebral aneurysm or aneurysmal SAH, or infratentorial siderosis. For further details, see ClinicalTrials. gov: NCT01856699. Patients primarily presented at stroke services $(n=194)$ or memory clinics $(n=108)$. The main clinical symptoms that led to presentation to the hospital were as follows: intracranial hemorrhage $(36 \%, 109 / 302)$, memory complaints $(36 \%, 108 / 302)$, ischemic stroke or TIA (14\%, 42/302), transient focal neurologic episodes or presumed focal seizure $11 \%(32 / 302)$, and dizziness, headache, or other nonspecific neurologic complaints (4\%, 11/302). Patients with CAA-related inflammation were not included.

\section{Baseline assessments}

Standardized assessments at baseline encompassed a medical history (including for vascular risk factors and medication use), a neurologic examination, MRI, genotyping for $A P O E$ carrier status, modified Rankin Scale (mRS), and Mini-Mental State Examination (MMSE).

MRI examinations were performed according to standardized imaging protocols on 3.0 or $1.5 \mathrm{~T}$ MRI scanners at baseline and at follow-up including the following sequences: diffusionweighted, fluid-attenuated inversion recovery, proton density-weighted, T1-weighted, T2*-weighted gradient echo, susceptibility-weighted imaging (SWI), and time-of-flight magnetic resonance angiography. $\mathrm{CMB}$ and macrohemorrhages were defined according to the Standards for Reporting Vascular Changes on Neuroimaging criteria. ${ }^{13}$ $\mathrm{CMB}$ were marked manually; lesion masks were normalized to Montreal Neurologic Institute standard space and rated automatically as described previously. ${ }^{14}$

\section{Exposure}

cSS was identified on T2*/SWI MRI by 2 independent expert raters (F.A.W. and J.L.) without disagreements. cSS was categorized into focal ( $\leq 3$ affected gyri) and disseminated $(>3$ affected gyri). ${ }^{3,12}$ Disagreements on the extent of cSS were resolved by consensus reads, which were necessary in $4 \%$ of cases $(5 / 121)$. 


\section{Follow-up assessments}

All patients were invited for personal visits at the local study site 6 and 12 months after baseline. Standardized assessments included a history of incident events, a neurologic examination, mRS, and neuroimaging. Stroke (ischemic and hemorrhagic) was defined by an acute focal neurologic deficit in combination with a corresponding new lesion detected by MRI or a CT scan. Patients unable or unwilling to undergo repeat MRI were offered CT imaging. Patients not returning to the study center were interviewed by telephone. Information on patients not reached by telephone was obtained by interview of relatives, private practitioners, and medical records (figure 1).

\section{Data management}

Data were entered into machine readable case report files (Teleform; Electric Paper, Lüneburg, Germany), ${ }^{15}$ which were processed by the coordinating center after centralized quality control (QC) by an independent data manager.

\section{Outcomes}

The predefined primary composite endpoint was the rate of stroke or death (defined as all-cause mortality) at follow-up. Secondary outcomes included (1) incident intracranial hemorrhage (ICH or sulcal SAH) as confirmed by MRI or native CT and (2) functional outcome as assessed by the $\mathrm{mRS}$ (range, 0 [no symptoms]-6 [death]). All clinical and imaging endpoints were assessed by board-certified neurologists and neuroradiologists, respectively.

\section{Statistical analysis}

All analyses were performed using R Core Team 2017, version 3.4.0 (R: A language and environment for statistical computing; R Foundation for Statistical Computing, Vienna, Austria; R-project.org/). Baseline statistics were calculated using Student $t$ tests or $\chi^{2}$ tests where appropriate. Dichotomized analyses were carried out using logistic regression. Kaplan-Meier analysis and the log-rank test were carried out using the $\mathrm{R}$ package survival. Time to event was recorded in days. Ordinal logistic regression with ordered $\mathrm{mRS}$ as an outcome variable were carried out using the $\mathrm{R}$ package VGAM. Proportional odds were tested using a likelihood ratio test. The proportional odds assumption of equal slopes was met in all models. To adjust for possible confounders, we applied backward stepwise regression analysis optimizing on Akaike Information Criterion to select the optimal set of covariates for the main model. As a result, the following baseline measures were included into all ordinal

Figure 1 Study flow chart

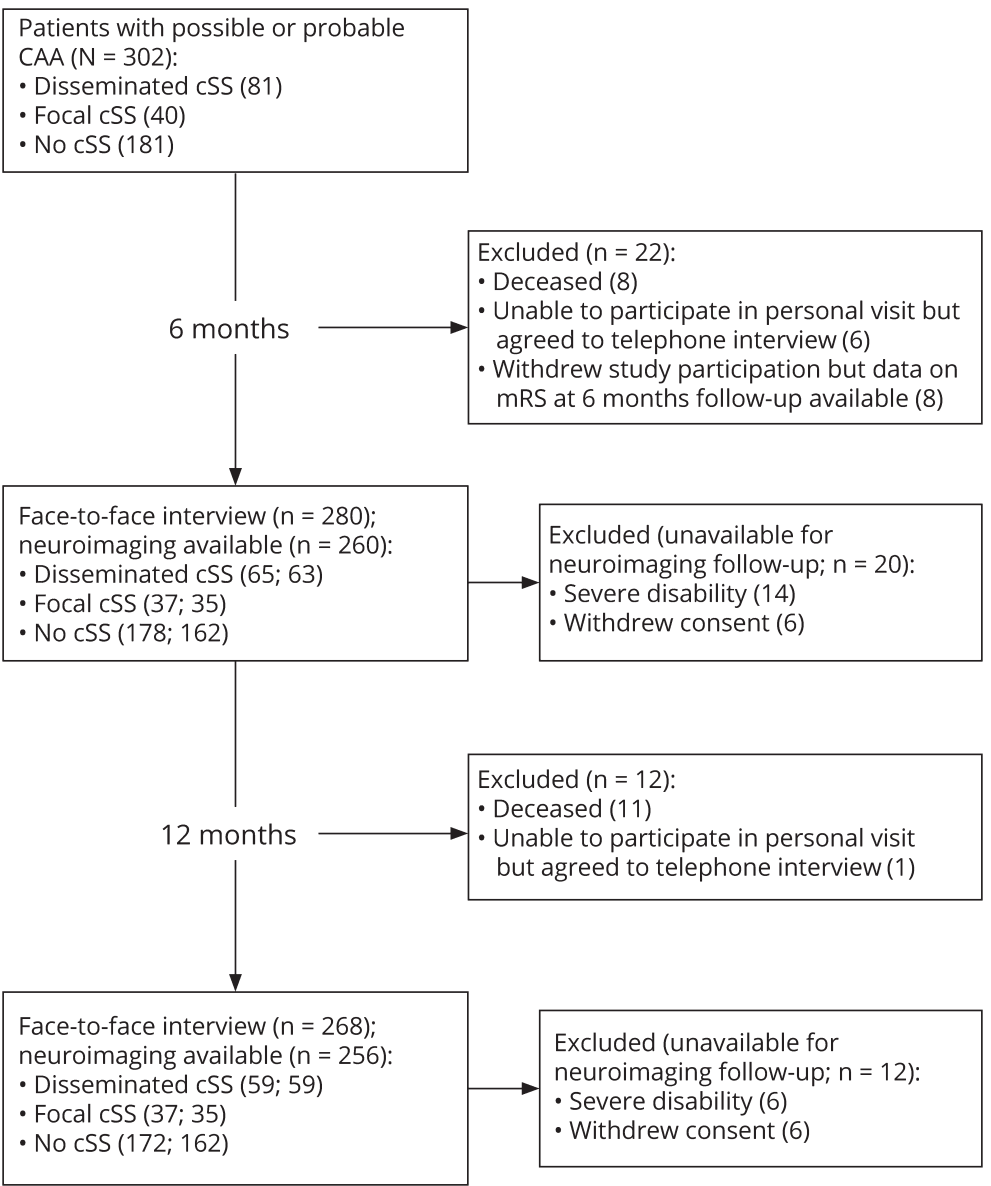

CAA = cerebral amyloid angiopathy; cSS = cortical superficial siderosis. 
logistic regression models: age, number of $\mathrm{CMB}$ at baseline, and $\mathrm{mRS}$ at baseline. The number of $\mathrm{CMB}$ was coded in $\mathrm{CMB}$ groups. Grouping into $0,1,2-4$, and $>5$ was carried out according to recent literature. ${ }^{16,17}$ Interaction between subgroups was tested using the rma function from metafor. We further present results for an extended model corrected for age, sex, hypertension, atrial fibrillation, use of antiplatelet agents, use of oral anticoagulants, number of $\mathrm{CMB}$ at baseline, previous intracranial hemorrhage (including both index events and a medical history of intracranial hemorrhage), previous ischemic stroke, and $\mathrm{mRS}$ at baseline.

\section{Data availability}

Any data not published within this article are available in the supplemental tables (doi:10.5061/dryad.rb9665d). Patientrelated data will be shared on request from any qualified investigator, maintaining anonymization of the individual patients.

\section{Standard protocol approvals, registrations, and patient consents}

Study approval was obtained from the institutional review boards at each site with informed consent from all participants. The study is registered at clinicaltrials.gov (NCT01856699).

\section{Results}

\section{Baseline characteristics}

Patients with cSS $(40 \%, 121 / 302)$ and without cSS $(60 \%$, $181 / 302$ ) were balanced with respect to age, sex, APOE carrier status, and hypertension (table 1). Thirty-six percent $(109 / 302)$ of patients had a history of previous intracranial hemorrhage, whereas 64\% (193/302) had lobar CMB or cSS without previous intracranial hemorrhage. Compared to patients without cSS, patients with cSS more often had a history of intracranial hemorrhage, and their MMSE scores and median mRS scores were lower. In contrast, patients without cSS more often had a history of hyperlipidemia, diabetes, ischemic stroke, and use of antiplatelet agents.

\section{Incident events and functional outcome stratified for the presence of cSS}

The primary composite endpoint of stroke or death of any cause occurred in $22 \%(27 / 121)$ of patients with cSS, as compared with $8 \%(15 / 181)$ of patients without cSS (table 2). In logistic regression analyses, the endpoint of stroke or death of any cause was reached significantly more frequently in patients with cSS compared to patients without cSS (adjusted odds ratio [OR] 1.2, 95\% confidence interval [CI] 1.1-1.3, $p=0.0005$, table 3). Incident intracranial hemorrhage occurred in $17 \%$ $(20 / 121)$ of patients with cSS and $4 \%(7 / 181)$ of patients without cSS. In logistic regression analyses, cSS was significantly associated with incident intracranial hemorrhage (adjusted OR $1.2,95 \%$ CI 1.1-1.3, $p=0.0003$ ). Death was significantly more frequent in patients with cSS $(11 \%, 13 / 121)$ than in patients without cSS $(3 \%, 6 / 181, p=0.01)$. In Kaplan-Meier analyses, the presence of cSS at baseline was a predictor of both faster time until the primary composite endpoint of stroke or death of any cause (log-rank test $p=0.0001$, figure $2 \mathrm{~A}$ ) and faster time until incident intracranial hemorrhage (log rank test: $p=0.00003$, figure 2B). Of note, incident intracranial hemorrhage accounted for the majority of primary endpoints in patients with cSS $(20 / 27 ; 74 \%)$ but not in patients without cSS $(7 / 15 ; 47 \%)$.

Functional independence (mRS 0-2) at the 1-year follow-up was observed in 59\% (71/121) of patients with cSS and in $82 \%(148 / 181)$ of patients without cSS (table 2 and figure 3 ). Corresponding rates for excellent outcome (mRS 0-1) were $45 \%(55 / 121)$ and $69 \%(125 / 181)$. In logistic regression analyses accounting for baseline variables including $\mathrm{mRS}$, both functional independence and excellent outcome were significantly less frequent in patients with cSS than in patients without cSS (adjusted OR 0.3, 95\% CI 0.1-0.8, $p=0.01$; and adjusted OR $0.3,95 \%$ CI $0.1-0.7, p=0.005$, respectively, table 3 ). The results remained significant in shift analyses using ordinal logistic regression after adjustment for possible confounders identified by backward stepwise regression (common OR 1.9, 95\% CI 1.2-3.1, $p=0.009$ ) and when forcing incident intracranial hemorrhage into the model (OR $1.8[1.0-2.8], p=0.03$ ). Results on the primary outcome, intracranial hemorrhage, and functional outcome further remained significant when extending the number of covariates in the logistic regression model to age, sex, hypertension, atrial fibrillation, use of antiplatelet agents, use of oral anticoagulants, number of CMB at baseline, previous intracranial hemorrhage, previous ischemic stroke, and $\mathrm{mRS}$ at baseline (table e-1, doi.org/10.5061/dryad.rb9665d).

\section{Incident events and functional outcome stratified for the extent of cSS}

cSS was disseminated in 67\% (81/121) and focal in 33\% (40/ 121). The primary composite endpoint occurred in $27 \%$ (22/ 81) of patients with disseminated cSS and in $12 \%(5 / 40)$ of patients with focal cSS ( $p=0.1$; table e-2 [doi.org/10.5061/ dryad.rb9665d]). Incident intracranial hemorrhage occurred in $20 \%(16 / 81)$ of patients with disseminated cSS and $10 \%$ $(4 / 40)$ of patients with focal cSS ( $p=0.2$; table e-2 [doi.org/ 10.5061/dryad.rb9665d]).

In logistic and ordinal logistic regression analyses, disseminated cSS was an independent predictor of reaching the primary composite endpoint (adjusted OR 5.0, 95\% CI 2.3-10.9, $p=0.00005$ ), intracranial hemorrhage (adjusted OR 6.7, 95\% CI 2.5-18.0, $p=0.0001$ ), and for unfavorable mRS scores at follow-up (common OR 2.6; 95\% CI 1.5-4.5, $p=0.0007$, table 3). In contrast, focal cSS was not a significant independent predictor of any of the outcomes in logistic and ordinal regression analyses.

\section{Incident events and functional outcome stratified for subgroups}

We next repeated the logistic and ordinal logistic regression analyses for the presence of cSS in the subgroup of patients with probable CAA without exclusion of patients with 1 or 2 deep CMB $(71 \%, 214 / 302)$. Results for the primary endpoint, intracranial hemorrhage, and functional outcome remained 
Table 1 Baseline characteristics in patients with suspected cerebral amyloid angiopathy stratified for presence of cortical superficial siderosis (CSS)

\begin{tabular}{|c|c|c|c|}
\hline & $\operatorname{cSS}(n=121)$ & No cSS $(n=181)$ & $p$ Value \\
\hline Age, y, mean (SD) & $73.80(6.91)$ & $73.15(7.84)$ & 0.5 \\
\hline Female, n (\%) & $52(43)$ & $69(38)$ & 0.5 \\
\hline$A P O E \varepsilon 2$ ( $\geq 1$ copy), $n(\%)^{a}$ & $20(17)$ & $23(13)$ & 0.4 \\
\hline$A P O E \varepsilon 4\left(\geq 1\right.$ copy), $n(\%)^{a}$ & $28(23)$ & $33(18)$ & 0.3 \\
\hline Hypertension, n (\%) & $87(72)$ & $142(78)$ & 0.2 \\
\hline Hyperlipidemia, n (\%) & $58(48)$ & $111(61)$ & 0.03 \\
\hline Diabetes, n (\%) & $13(11)$ & $47(26)$ & 0.002 \\
\hline Coronary artery disease, $\mathbf{n}(\%)$ & $9(7)$ & $25(14)$ & 0.1 \\
\hline Previous myocardial infarction, n (\%) & $6(5)$ & $21(12)$ & 0.08 \\
\hline Peripheral artery disease, n (\%) & $12(10)$ & $34(19)$ & 0.05 \\
\hline Atrial fibrillation, n (\%) & $17(14)$ & $36(20)$ & 0.3 \\
\hline Inclusion from stroke service & $72(60)$ & $122(67)$ & 0.2 \\
\hline Inclusion from memory clinic & $49(41)$ & $59(33)$ & 0.2 \\
\hline Previous TIA, $n(\%)^{b}$ & $12(10)$ & $30(17)$ & 0.1 \\
\hline Previous ischemic stroke, $\mathrm{n}(\%)^{\mathrm{b}}$ & $25(21)$ & $92(51)$ & $<0.0001$ \\
\hline Months since previous ischemic stroke median (Q3-Q1) & $14(1-34)$ & $2(1-30)$ & 0.5 \\
\hline Previous intracranial hemorrhage, $n(\%)^{b}$ & $71(59)$ & $38(21)$ & $<0.0001$ \\
\hline Intracerebral hemorrhage, $\mathrm{n}(\%)$ & $50(41)$ & $38(21)$ & 0.0002 \\
\hline Sulcal subarachnoid hemorrhage, $n$ (\%) & $35(29)$ & $4(2)$ & $<0.0001$ \\
\hline Months since previous intracranial hemorrhage (median Q3-Q1) & $4(1-16)$ & $7(1-40)$ & 0.4 \\
\hline Cerebral microbleeds, $\mathrm{n}(\%)^{\mathrm{c}}$ & & & 0.007 \\
\hline 0 & $28(24)$ & $22(12)$ & \\
\hline 1 & $15(13)$ & $29(16)$ & \\
\hline $2-4$ & $27(23)$ & $67(38)$ & \\
\hline$\geq 5$ & $49(41)$ & $60(34)$ & \\
\hline Antiplatelet agents, $\mathrm{n}(\%)$ & $34(28)$ & $104(57)$ & $<0.0001$ \\
\hline Oral anticoagulation, $\mathrm{n}(\%)$ & $14(12)$ & $23(13)$ & 0.9 \\
\hline Mini-Mental State Examination, mean (SD) & $24.2(5.6)$ & $25.8(4.4)$ & 0.009 \\
\hline \multicolumn{4}{|l|}{ Functional outcome } \\
\hline mRS, median (Q3-Q1) & $1(2-0)$ & $1(2-0)$ & 0.02 \\
\hline mRS 0-1, n (\%) & $73(60)$ & $127(70)$ & 0.07 \\
\hline mRS 0-2, n (\%) & $94(78)$ & $156(86)$ & 0.07 \\
\hline
\end{tabular}

Abbreviation: $\mathrm{mRS}=$ modified Rankin Scale.

a Missing, $n=22$ (no consent for molecular genetics).

${ }^{\mathrm{b}}$ Definition, both index events and events determined by medical history.

${ }^{c}$ Missing, $n=5$ (because of motion artefacts or other technical issues).

significant (figure 4). We further repeated the analysis in the subgroup of patients with probable CAA and strictly lobar CMB (i.e., with exclusion of all patients with any deep CMB, $59 \%, 179 / 302)$ thus fulfilling the modified Boston criteria. ${ }^{12}$
Again, the results for the primary endpoint, intracranial hemorrhage, and functional outcome all remained significant (table e-4, doi.org/10.5061/dryad.rb9665d), thus confirming the results of the overall cohort. 
Table 2 Incident events and functional outcome in patients with suspected cerebral amyloid angiopathy stratified for presence of cortical superficial siderosis (cSS)

\begin{tabular}{llll}
\hline & $\begin{array}{l}\text { cSS } \\
(\mathbf{n}=\mathbf{1 2 1})\end{array}$ & $\begin{array}{l}\text { No cSS } \\
(\mathbf{n}=\mathbf{1 8 1})\end{array}$ & $\boldsymbol{p}$ Value \\
\hline Stroke or death, $\mathbf{n}(\%)$ & $27(22)$ & $15(8)$ & 0.001 \\
\hline Ischemic events, $\mathbf{n}(\%)$ & $3(2)$ & $10(6)$ & 0.3 \\
\hline Ischemic stroke & $1(1)$ & $7(4)$ & 0.2 \\
\hline TIA & $2(2)$ & $3(2)$ & 1 \\
\hline Intracranial hemorrhage, $\mathbf{n}(\%)$ & $20(17)$ & $7(4)$ & 0.0003 \\
\hline Intracerebral hemorrhage & $17(14)$ & $7(4)$ & 0.002 \\
\hline Sulcal subarachnoid hemorrhage & $3(2)$ & 0 & 0.06 \\
\hline Death, $\mathbf{n}(\%)$ & $13(11)^{\mathrm{a}}$ & $6(3)^{\mathrm{b}}$ & 0.01 \\
\hline
\end{tabular}

Functional outcome

\begin{tabular}{llll}
\hline mRS 0-1, n (\%) & $55(45)$ & $125(69)$ & 0.00005 \\
\hline mRS 0-2, n (\%) & $71(59)$ & $148(82)$ & 0.00002
\end{tabular}

Abbreviation: $\mathrm{mRS}=$ modified Rankin Scale.

${ }^{\text {a }}$ Causes of death: imaging confirmed intracranial hemorrhage (7 patients) sudden death of presumed vascular cause $(n=5)$, pulmonary embolism (1 patient).

${ }^{b}$ Causes of death: imaging confirmed intracranial hemorrhage (1 patient) ischemic stroke (1 patient), pneumonia (3 patients), pancreatic cancer (1 patient).

Next, we repeated the analysis for the subgroups of patients recruited through stroke services $(64 \%, 194 / 302)$ and memory clinics $(36 \%, 108 / 302)$. In logistic regression analyses, results for the primary endpoint, intracranial hemorrhage, and functional outcome reached statistical significance in patients recruited via stroke services but not in patients recruited through memory clinics (figure 4), although the test for interaction between group and cSS was not significant.

\section{Incident events and functional outcome} stratified for small vessel disease (SVD) severity We further examined the predictive ability of a recently proposed CAA-based SVD score ${ }^{17}$ that includes cSS in addition to the presence and extent of other MRI features (white matter hyperintensities [WMH], perivascular spaces [PVS], and CMB). In logistic and ordinal logistic regression analyses the total score predicted the primary endpoint (adjusted OR 1.2, 95\% CI $1.1-1.3, p=0.001$ ), intracranial hemorrhage (adjusted OR 1.2, 95\% CI 1.1-1.3, $p=0.0003$ ), and unfavorable $\mathrm{mRS}$ scores at follow-up (common OR 1.3, 95\% CI 1.1-1.6, $p=0.0009$ ). Of note, however, cSS was the only MRI marker that predicted the primary outcome or intracranial hemorrhage when considered in isolation (table e-3, doi.org/10.5061/dryad.rb9665d).

\section{Discussion}

The main findings from this prospective multicenter study on a large cohort of patients with probable or possible CAA can
Table 3 Incident events and functional outcome in logistic and ordinal logistic regression analysis stratified for presence and extent of cortical superficial siderosis (CSS)

\begin{tabular}{llll}
\hline & $\begin{array}{l}\text { Presence of } \\
\text { cSS }(\mathbf{n}=\mathbf{1 2 1})\end{array}$ & $\begin{array}{l}\text { Disseminated } \\
\text { cSS }(\mathbf{n}=\mathbf{8 1})\end{array}$ & $\begin{array}{l}\text { Focal cSS } \\
(\mathbf{n}=\mathbf{4 0})\end{array}$ \\
\hline Stroke or death & & & \\
\hline OR $(95 \% \mathrm{Cl})$ & $1.2(1.1-1.3)$ & $5.0(2.3-10.9)$ & $1.4(0.4-4.5)$ \\
\hline $\boldsymbol{p}$ Value & 0.0005 & 0.00005 & 0.6 \\
\hline $\begin{array}{l}\text { Intracranial } \\
\text { hemorrhage }\end{array}$ & & & \\
\hline OR (95\% CI) & $1.2(1.1-.3)$ & $6.7(2.5-18.0)$ & $2.3(0.6-8.8)$ \\
\hline $\boldsymbol{p}$ Value & 0.0003 & 0.0001 & 0.2 \\
\hline
\end{tabular}

\section{Functional}

outcome

\begin{tabular}{llll}
\hline $\begin{array}{c}\text { mRS, shift } \\
\text { analysis, OR } \\
(\mathbf{9 5 \%} \mathrm{CI})\end{array}$ & $1.9(1.2-3.1)$ & $2.6(1.5-4.5)$ & $1.1(0.5-2.2)$ \\
\hline $\boldsymbol{p}$ Value & 0.009 & 0.0007 & 0.8 \\
\hline
\end{tabular}

mRS 0-1

\begin{tabular}{llll}
\hline OR $(95 \% \mathrm{CI})$ & $0.3(0.1-0.7)$ & $0.2(0.1-0.6)$ & $1.1(0.3-3.3)$ \\
\hline $\boldsymbol{p}$ Value & 0.005 & 0.002 & 0.9
\end{tabular}

mRS 0-2

\begin{tabular}{llll}
\hline OR $(95 \% \mathrm{CI})$ & $0.3(0.1-0.8)$ & $0.2(0.1-0.6)$ & $1.4(0.4-5)$ \\
\hline $\boldsymbol{p}$ Value & 0.01 & 0.001 & 0.6 \\
\hline
\end{tabular}

Abbreviations: $\mathrm{Cl}=$ confidence interval; diss $=$ disseminated $(>3$ gyri affected); $m R S=$ modified Rankin Scale; $O R=$ odds ratio.

Covariates selected by stepwise regression: age, number of cerebral microbleeds at baseline, and mRS at baseline. Models adjusted for age, sex, hypertension, atrial fibrillation, intake of platelet inhibitors, intake of oral anticoagulation, presence of cerebral microbleeds, history of intracranial hemorrhage and ischemic stroke, and mRS at baseline.

be summarized as follows: (1) both the presence and extent of cSS are strong predictors of the predefined composite endpoint of stroke or death within 12 months from baseline; (2) incidence rates for intracranial hemorrhage were remarkably high in CAA patients with cSS (17\%) compared to those without cSS (4\%); and (3) the presence and extent of cSS predict an unfavorable functional outcome.

The incidence rate for the primary composite endpoint of stroke or death within 12 months from baseline was high, reaching $22 \%$ in patients with cSS compared to $8 \%$ in patients without cSS. Importantly, all but 1 of incident strokes in patients with cSS were classified as CAA-related intracranial hemorrhage (defined as $\mathrm{ICH}$ or sulcal SAH), emphasizing the role of cSS as a predictor for intracranial hemorrhage risk. Mortality was significantly higher in patients with cSS compared with patients without cSS, and in $92 \%$ of the patients with cSS who died during follow-up, the underlying cause was intracranial hemorrhage or sudden death of presumed 
Figure 2 Kaplan-Meier analyses in patients with suspected cerebral amyloid angiopathy stratified for the presence of cortical superficial siderosis (cSS)
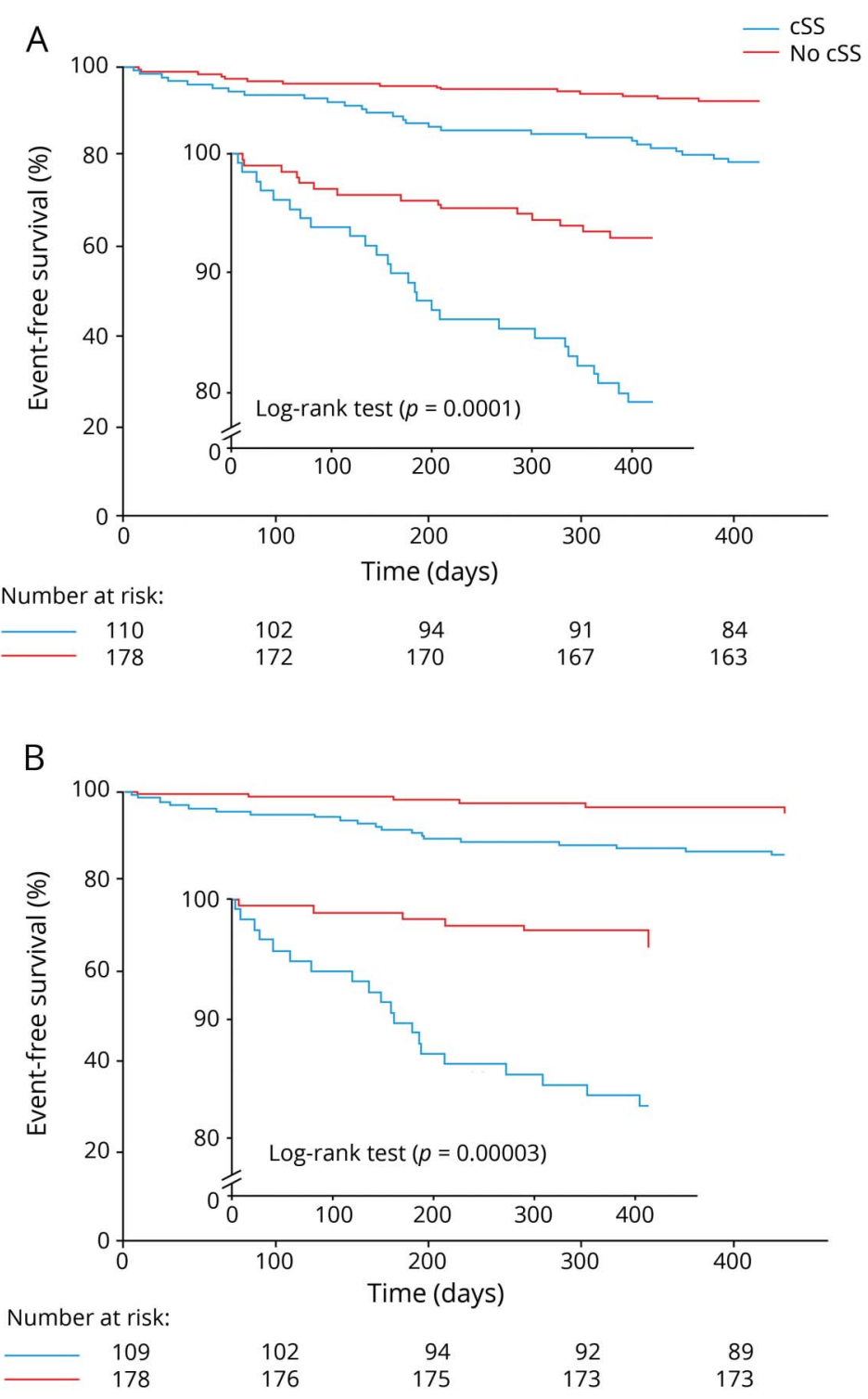

vascular cause. These observations highlight the prognostic relevance of cSS in patients with probable or possible CAA, particularly when viewed in conjunction with our data on functional outcome.

We found the presence of cSS to be associated with worse functional outcome 12 months from baseline both in binary analyses using different cutoffs and in shift analyses using ordinal logistic regression. While somewhat expected from the higher rate of intracranial hemorrhage, this finding emphasizes the clinical relevance of cSS, for which data on functional outcome have so far been missing. On average, patients with cSS were functionally more impaired at baseline than patients without cSS. Importantly, however, differences in outcomes remained significant both in the main logistic regression analyses controlling for functional status at baseline among other factors and after forcing incident intracranial hemorrhage into the model. Hence, our study establishes cSS as an independent risk factor for poor functional outcome.

The proportion of patients who experienced an ICH within 12 months from baseline was $14 \%$ in patients with cSS and $4 \%$ in those without cSS. A recent study in survivors of spontaneous symptomatic probable or possible CAA-related lobar ICH found corresponding figures of $16 \%$ and $5 \%$ within a 6-month interval. ${ }^{18}$ Differences in incidence rates for ICH likely reflect differences in case mix, study protocols, and possibly also medication use. For instance, only $29 \%$ of our patients had a previous $\mathrm{ICH}$ and the average age in our cohort was slightly lower than in the study on ICH survivors. ${ }^{18}$ Regardless of these differences, the 2 studies underscore the clinical importance of cSS in predicting $\mathrm{ICH}$ risk in patients with probable or possible CAA. 
Figure 3 Distribution of modified Rankin Scale scores at baseline and at 12-month follow-up stratified for the presence of cortical superficial siderosis (cSS)

A

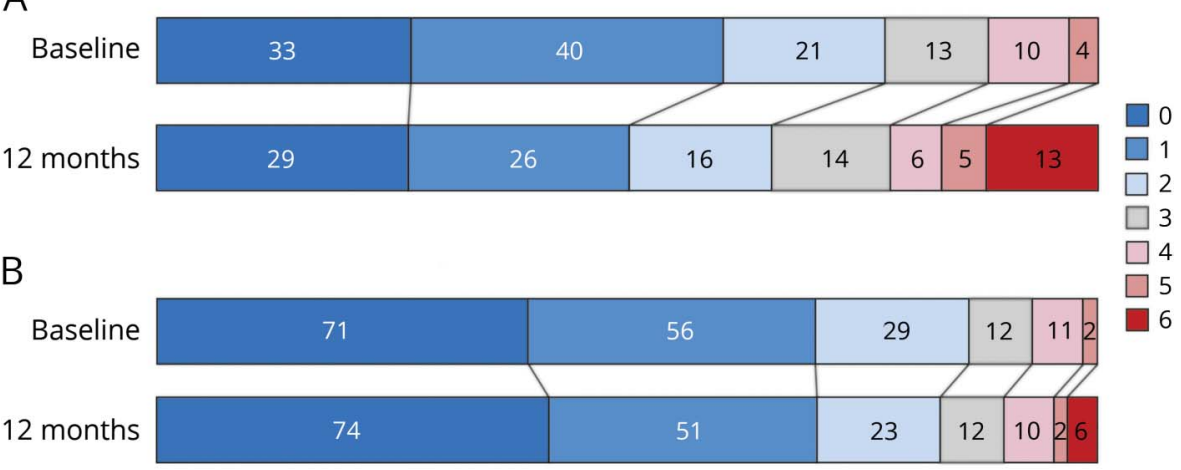

(A) Cerebral amyloid angiopathy (CAA) with cSS. (B) CAA without cSS.

A previous study in $\mathrm{ICH}$ survivors found a higher recurrence rate of ICH in patients with disseminated cSS when compared to patients with focal cSS. ${ }^{18}$ Another study in ICH survivors found disseminated cSS, but not the presence of cSS, to be associated with recurrent $\mathrm{ICH} .{ }^{19}$ We found both the presence of cSS and disseminated cSS to be independent predictors (table 3) for the primary composite endpoint, for intracranial hemorrhage, and for an unfavorable mRS, whereas the ORs for focal cSS did not reach statistical significance. However, the number of patients with focal cSS was relatively small $(\mathrm{n}=$ 40), leaving the possibility that we have missed a possible effect of focal cSS on the primary and secondary endpoints because of a lack in statistical power.

Thirty-six percent of our patients were recruited through memory clinics. Focusing on this subgroup, the presence of cSS was not a significant predictor of incident events. However, since the interaction test for group and cSS was not significant, the cSS-associated risk is probably similar between stroke service and memory clinic patients, but with lower confidence in the latter group due to smaller sample size. Additional studies are needed to determine the precise risk of incident events and functional outcome in CAA patients who present with cognitive complaints.

We found a recently proposed CAA-based SVD score ${ }^{17}$ that includes cSS in addition to the presence and extent of $\mathrm{WMH}$, PVS, and CMB to predict both the primary endpoint and the secondary endpoints of intracranial hemorrhage and functional outcome. We did not formally compare the predictive value of the composite score with cSS alone as this was outside the scope of our study. Of note, however, cSS was the only MRI marker that predicted outcome when considered in isolation (table e-3, doi.org/10.5061/dryad.rb9665d). More work is needed to determine whether the use of the composite score adds to prognostication in CAA when compared to the cSS alone.

The proportion of patients taking antiplatelet agents was significantly higher among those without cSS than those with
cSS. Still, incidence rates for intracranial hemorrhage were substantially higher in the latter group. While the current study was not designed to test the effects of medication use on vascular risk, our findings highlight the need for studying the effects of antithrombotic use on incident cerebrovascular events in CAA patients while also stratifying for cSS.

Strengths of this study include the prospective setting with systematic follow-up by personal visits and neuroimaging. The retention rate was high with $93 \%$ and $89 \%$ of the patients returning for the face-to-face interviews at 6 and 12 months, respectively. Also, data on incident stroke and functional outcome were available for all 302 patients. Hence, information on the primary endpoint and on functional outcome was complete. Our study also has limitations. Despite a rather large sample size and a follow-up interval of 12 months, the total number of events for the primary endpoint was limited $(n=42)$. To restrict the analyses to a meaningful number of covariates, we applied a stepwise regression model. While we cannot rule out residual confounding with the selected covariates, the results remained significant in an extended model adjusting for 9 covariates selected on the basis of previous literature (table e-1, doi.org/10.5061/dryad. rb9665d). Finally, we did not exclude patients with a maximum of 2 deep CMB provided they had other typical CAA markers. Importantly, however, the results remained significant when excluding patients with any deep CMB (20\% of our cohort, 59/302).

Follow-up MRI could not be obtained in 50\% of patients. However, any imaging follow-up (MRI or CT) was available in $85 \%$ of the overall cohort and in all patients who experienced an incident stroke. Therefore, key secondary outcomes including intracranial hemorrhage were fully captured. The recruitment of patients from different centers may have resulted in slightly variable assessments. However, variations in assessments were minimized by a detailed study protocol, central data management, and rigorous QC. The use of 2 different field strengths (1.5 and 3.0T) might have influenced 
Figure 4 Subgroup analyses of incident events and functional outcome in logistic and ordinal logistic regression stratified for the presence of cortical superficial siderosis (CSS)
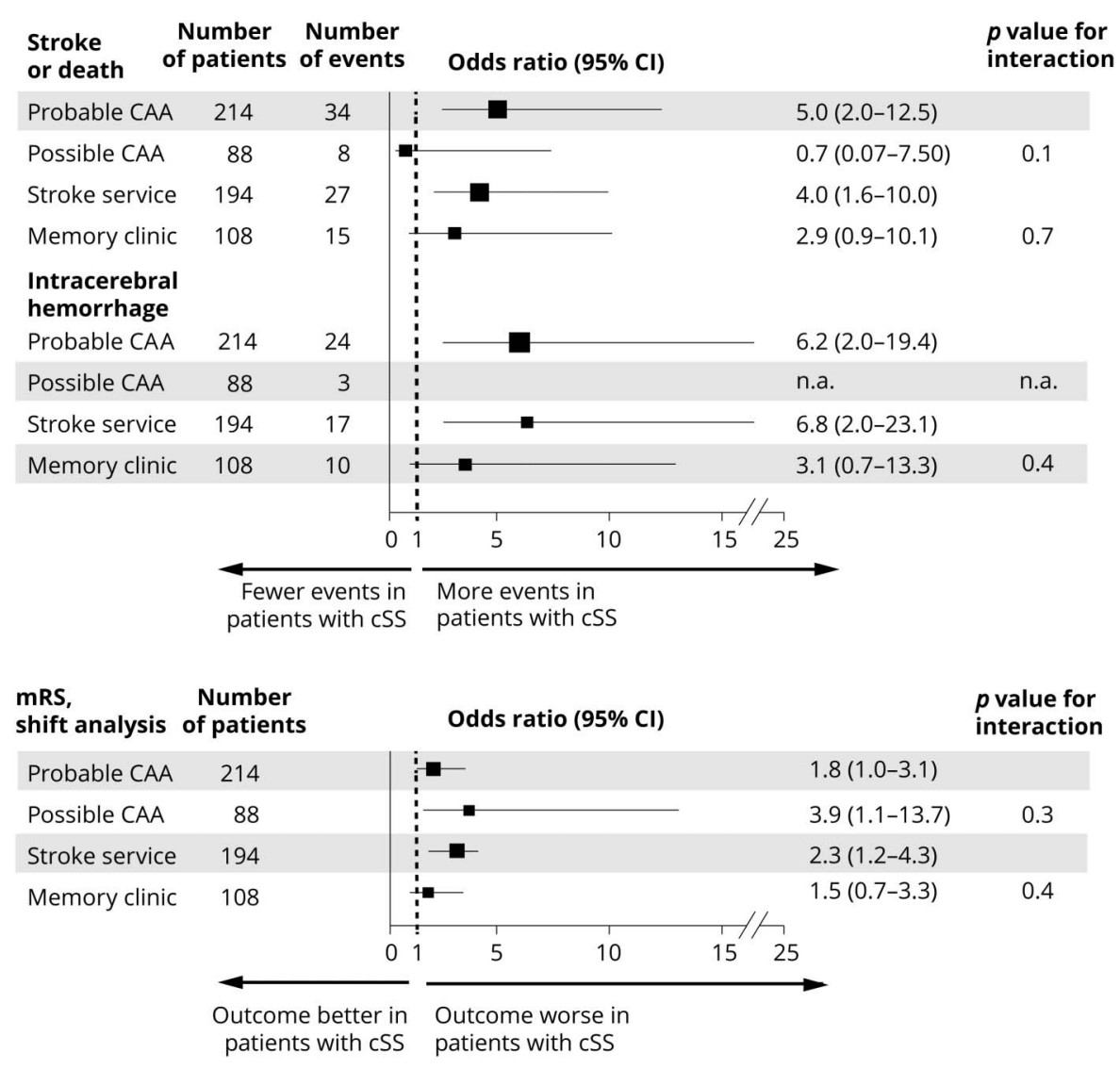

$\mathrm{CAA}=$ cerebral amyloid angiopathy; $\mathrm{Cl}=$ confidence interval.

the accuracy of the detection and extent of cSS and CMB counts. However, MRI field strength as a variable showed no significant association with our endpoints in univariate analysis and was not selected by stepwise regression models, arguing against a strong effect. Also, the multicenter approach otherwise enhances the generalizability of our findings.

The current study shows that the presence of cSS strongly predicts the composite outcome of stroke or death of any cause with a high rate of incident intracranial hemorrhage over 12 months from baseline particularly in patients with disseminated cSS. We further found cSS to be associated with a higher mortality and with poor outcome. Our findings have clinical relevance. The exceedingly high rate of intracranial hemorrhage and associated mortality and risk of poor functional outcome in patients with cSS requires attention, particularly when balancing the risks and benefits of antithrombotic treatment in patients with probable or possible CAA. However, the optimal therapeutic strategy will eventually need to be determined in a controlled trial.

\section{Author contributions}

Frank Arne Wollenweber: study concept and design, acquisition of data, manuscript writing. Christian Opherk: study concept and design, analysis and interpretation of data. Marialuisa Zedde: acquisition of data, analysis and interpretation of data. Cihan Catak: acquisition of data, analysis and interpretation of data. Rainer Malik: analysis and interpretation of data. Marco Duering: acquisition of data, analysis and interpretation of data. Marek-Janusz Konieczny: acquisition of data, analysis and interpretation of data. Rosario Pascarella: analysis and interpretation of data. Raquel Samões: analysis and interpretation of data. Manuel Correia: analysis and interpretation of data. Joan Martí-Fàbregas: analysis and interpretation of data. Jennifer Linn: analysis and interpretation of data. Martin Dichgans: study concept and design, manuscript writing.

\section{Study funding}

Deutsche Forschungsgemeinschaft (German Research Foundation), Munich Cluster for Systems Neurology (EXC 1010 SyNergy), and European Union's Horizon 2020 research and innovation programmes under grant agreements no. 666881 (SVDs@target) and 667375 (CoSTREAM).

\section{Disclosure}

The authors report no disclosures relevant to the manuscript.Go to Neurology.org/ $\mathrm{N}$ for full disclosures. 


\section{Publication history}

Received by Neurology May 9, 2018. Accepted in final form October 16, 2018.

\section{References}

1. Wollenweber FA, Opherk C, Zedde M, et al. European Stroke Organisation, conference: abstracts. Eur Stroke J 2018;3:3-586.

2. Arvanitakis Z, Leurgans SE, Wang Z, Wilson RS, Bennett DA, Schneider JA. Cerebra amyloid angiopathy pathology and cognitive domains in older persons. Ann Neurol 2011;69:320-327.

3. Charidimou A, Boulouis G, Gurol ME, et al. Emerging concepts in sporadic cerebral amyloid angiopathy. Brain 2017;140:1829-1850.

4. Moulin S, Labreuche J, Bombois S, et al. Dementia risk after spontaneous intracerebral haemorrhage: a prospective cohort study. Lancet Neurol 2016;15:820-829.

5. Kase CS, Williams JP, Wyatt DA, Mohr JP. Lobar intracerebral hematomas: clinical and CT analysis of 22 cases. Neurology 1982;32:1146-1150.

6. Massaro AR, Sacco RL, Mohr JP, et al. Clinical discriminators of lobar and deep hemorrhages: the Stroke Data Bank. Neurology 1991;41:1881-1885.

7. O'Donnell HC, Rosand J, Knudsen KA, et al. Apolipoprotein E genotype and the risk of recurrent lobar intracerebral hemorrhage. N Engl J Med 2000;342:240-245.

8. DeSimone CV, Graff-Radford J, El-Harasis MA, Rabinstein AA, Asirvatham SJ, Holmes DR Jr. Cerebral amyloid angiopathy and implications for atrial fibrillation management. Lancet 2017;390:9-11.

9. Charidimou A, Boulouis G, Xiong L, et al. Cortical superficial siderosis and first-ever cerebral hemorrhage in cerebral amyloid angiopathy. Neurology 2017;88:1607-1614.
10. Charidimou A, Peeters AP, Jäger R, et al. Cortical superficial siderosis and in tracerebral hemorrhage risk in cerebral amyloid angiopathy. Neurology 2013;81: $1666-1673$.

11. Linn J, Wollenweber FA, Lummel N, et al. Superficial siderosis is a warning sign for future intracranial hemorrhage. J Neurol 2013;260:176-181.

12. Linn J, Halpin A, Demaerel P, et al. Prevalence of superficial siderosis in patients with cerebral amyloid angiopathy. Neurology 2010;74:1346-1350.

13. Wardlaw JM, Smith EE, Biessels GJ, et al. Neuroimaging standards for research into small vessel disease and its contribution to ageing and neurodegeneration. Lance Neurol 2013;12:822-838.

14. Wollenweber FA, Baykara E, Zedde M, et al. Cortical superficial siderosis in different types of cerebral small vessel disease. Stroke 2017;48:1404-1407.

15. Jenkins TM, Wilson Boyce T, Akers R, et al. Evaluation of a Teleform-based data collection system: a multi-center obesity research case study. Comput Biol Med 2014;49:15-18.

16. Charidimou A, Boulouis G, Roongpiboonsopit D, et al. Cortical superficial siderosis multifocality in cerebral amyloid angiopathy: a prospective study. Neurology 2017;89: 2128-2135.

17. Charidimou A, Martinez-Ramirez S, Reijmer YD, et al. Total magnetic reso nance imaging burden of small vessel disease in cerebral amyloid angiopathy: an imaging-pathologic study of concept validation. JAMA Neurol 2016;73: 994-1001.

18. Roongpiboonsopit D, Charidimou A, William CM, et al. Cortical superficial siderosis predicts early recurrent lobar hemorrhage. Neurology 2016;87:1863-1870.

19. Moulin S, Casolla B, Kuchcinski G, et al. Cortical superficial siderosis: a prospective observational cohort study. Neurology 2018;91:e132-e138. 


\section{Neurology}

\section{Prognostic relevance of cortical superficial siderosis in cerebral amyloid angiopathy}

Frank Arne Wollenweber, Christian Opherk, Marialuisa Zedde, et al.

Neurology 2019;92;e792-e801 Published Online before print January 23, 2019

DOI 10.1212/WNL.0000000000006956

This information is current as of January 23, 2019

Updated Information \&
Services
References
Citations
Subspecialty Collections

Permissions \& Licensing

Reprints including high resolution figures, can be found at: http://n.neurology.org/content/92/8/e792.full

This article cites 19 articles, 9 of which you can access for free at: http://n.neurology.org/content/92/8/e792.full\#ref-list-1

This article has been cited by 4 HighWire-hosted articles: http://n.neurology.org/content/92/8/e792.full\#\#otherarticles

This article, along with others on similar topics, appears in the following collection(s):

All Cerebrovascular disease/Stroke

http://n.neurology.org/cgi/collection/all_cerebrovascular_disease_strok e

Intracerebral hemorrhage

http://n.neurology.org/cgi/collection/intracerebral_hemorrhage

MRI

http://n.neurology.org/cgi/collection/mri

Prognosis

http://n.neurology.org/cgi/collection/prognosis

Information about reproducing this article in parts (figures,tables) or in its entirety can be found online at:

http://www.neurology.org/about/about_the_journal\#permissions

Information about ordering reprints can be found online:

http://n.neurology.org/subscribers/advertise

Neurology ${ }^{\circledR}$ is the official journal of the American Academy of Neurology. Published continuously since 1951, it is now a weekly with 48 issues per year. Copyright (O 2019 American Academy of Neurology. All rights reserved. Print ISSN: 0028-3878. Online ISSN: 1526-632X.

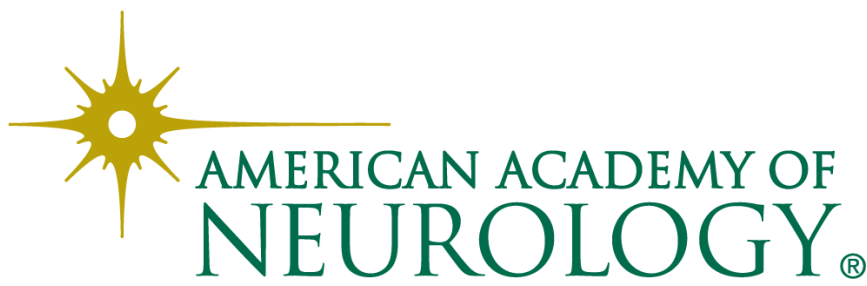

